\title{
A Computational Study for the Graph-Theoretic Version of the Union-Closed Sets Conjecture
}

\author{
M. I. Moussa \\ Computer Science Department, Faculty of Computers \& \\ Information, Benha University, Benha, Egypt,
}

\author{
E. M. Badr \\ Department of scientific computing, Faculty of Computers \\ \& Information, Benha University, Benha, Egypt,
}

\begin{abstract}
An induced subgraph $S$ of a graph $G$ is called a derived subgraph of $G$ if $S$ contains no isolated vertices. An edge $e$ of $G$ is said to be residual if $e$ occurs in more than half of the derived subgraphs of $G$. We prove some theorems which calculate the number of derived subgraphs for some special graphs. We also present a new algorithm SDSA that calculates the number of derived subgraphs for a given graph $G$ and determines the residual and non-residual edges. Finally, we introduce a computational study which supports our results.
\end{abstract}

\section{Keywords}

Union closed sets conjecture, induced graphs, derived subgraphs.

\section{INTRODUCTION}

A union-closed family of sets $\mathscr{A}$ is a finite collection of sets not all empty such that the union of any two members of $\mathscr{A}$ is also a member of $\mathscr{A}$. The following Conjecture is due to Peter Frankl $[1,2,3]$.

Conjecture 1. Let $\mathscr{A}=\left\{A_{1}, A_{2}, \ldots, A_{n}\right\}$ be a union-closed family of $n$ distinct sets. Then there exists an element which belongs to at least $n / 2$ of the sets in $\mathcal{A}$.

Let $A=\cup A_{i}$. If we replace each set $A_{i}$ by $B_{i}=A-A_{i}$ then we get an intersection-closed family of sets, which we call the dual family of $A$. Therefore Conjecture 1 is equivalent to the following.

Conjecture 2. Let $B=\left\{B_{1}, B_{2}, \ldots, B_{n}\right\}$ be an intersectionclosed family of $n$ distinct sets. Then there exists an element which belongs to at most $n / 2$ of the sets in $B$.

An induced subgraph $S$ of a graph $G$ is called a derived subgraph of $G$ if $S$ contains no isolated vertices. An edge $e$ of $G$ is said to be residual if $e$ occurs in more than half of the derived subgraphs of $G$ otherwise $e$ is non-residual. Let $D(G)$ denote the set of derived subgraphs of $G$ and put $n_{d}(G)=$ $|D(G)|$. A graph-theoretic version of the union-closed sets conjecture due to El-Zahar [4]. He formulated a weaker version of Conjecture 1 specialized for graphs as the following.

Conjecture 3. Every non-empty graph contains a nonresidual edge.

Example 1. The derived subgraphs of $C_{6}$ are $\varnothing, C_{6}$ and the subgraphs $S_{1}, S_{2}, \ldots, S_{5}$ together with their cyclic permutations as shown in Figure 1. In all, we have $n_{d}\left(C_{6}\right)=$ 29 ( compare to 64 induced subgraphs of $C_{6}$ ). Each edge of $C_{6}$ is contained in exactly 12 derived subgraphs and, therefore, is non-residual.

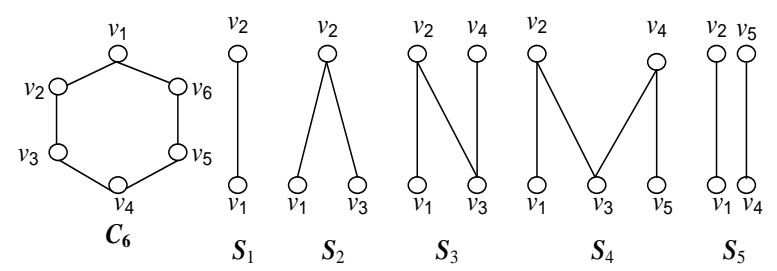

Fig.1: Derived Subgraphs of $C_{6}$

Example 2. Consider the graph $G_{1}$ of Figure 2. This graph has $n_{d}\left(G_{1}\right)=34$. Each of the edges $e_{1}, e_{2}, e_{3}$ occur in 18 derived subgraphs so that it is residual. The remaining edges are nonresidual belonging only to 13 derived subgraphs.

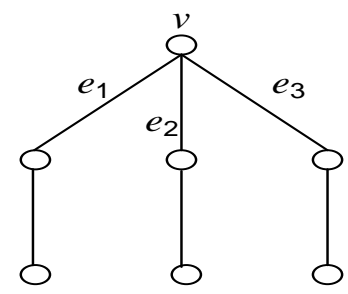

Fig. 2: Residual Edges

B. Llano et al proved that the every simple graph with at least one edge contains a non-residual edge (Conjecture 3) [5].

In this work, our aim is to introduce a computational study for derived subgraphs.

In Section 2, we prove some theorems which calculate the number of derived subgraphs for some special graphs. In Section 3, we present a new algorithm SDSA that calculates the number derived subgraphs for a given graph $G$ and determines the residual and non-residual edges. In Section 4 we give a computational study which supports our results. Finally, we give our conclusions and discuss possible extensions of the algorithm.

\section{THE MAIN RESULTS}

In this Section, we prove some theorems which calculate the number of derived subgraphs for some special graphs using the Principle of Mathematical Induction and the Principle of Inclusion-Exclusion. 
Theorem 1. Let $P_{n}$ be the path $v_{1}, v_{2}, v_{3}, \ldots, v_{n}$. Then the number of derived subgraphs of $P_{n}$ is given by the following relation :

$$
n_{d}\left(P_{n}\right)=\left\{\begin{array}{lr}
1 & \text { if } n=0,1 \\
2 & \text { if } n=2 \\
2 n_{d}\left(P_{n-1}\right)-n_{d}\left(P_{n-2}\right)+n_{d}\left(P_{n-3}\right) & \text { if } n \geq 3
\end{array}\right.
$$

Proof. We prove this theorem by the Principle of Mathematical Induction. The proof will now proceed in two steps : the initial step and the inductive step.

Initial Step. It is clear that the statement (1) is true for $n=0$, $1,2$.

Inductive Step. Here we must prove that the following assertion: " If there is a positive integer $k$ such that $n_{d}\left(P_{k}\right)=$ $2 n_{d}\left(P_{k-1}\right)-n_{d}\left(P_{k-2}\right)+n_{d}\left(P_{k-3}\right) \quad$ is true then $n_{d}\left(P_{k+1}\right)=2 n_{d}\left(P_{k}\right)-n_{d}\left(P_{k-1}\right)+n_{d}\left(P_{k-2}\right)$ is true. Thus, we assume there is a positive integer $k$ such that

$n_{d}\left(P_{k}\right)=2 n_{d}\left(P_{k-1}\right)-n_{d}\left(P_{k-2}\right)+n_{d}\left(P_{k-3}\right)$

Since $n_{d}\left(P_{k}\right)$ is the number of all derived subgraphs of $P_{k}$ so by adding one vertex $v_{k+1}$ to the relation ( 2 ), we will $n_{d}\left(P_{k+1}\right)$ as follows:

$n_{d}\left(P_{k} \cup\left\{v_{k+1}\right\}\right)=$

$2 n_{d}\left(P_{k-1} \cup\left\{v_{k+1}\right\}\right)-n_{d}\left(P_{k-2} \cup\left\{v_{k+1}\right\}\right)+n_{d}\left(P_{k-3} \cup\left\{v_{k+1}\right\}\right)$

then $n_{d}\left(P_{k+1}\right)=2 n_{d}\left(P_{k}\right)-n_{d}\left(P_{k-1}\right)+n_{d}\left(P_{k-2}\right)$ so the relation (1) is true for $n \geq 0$.

Theorem 2 . Let $C_{n}$ be a cycle on $n \geq 3$ vertices. Then the number of derived subgraphs of $C_{n}$ is given by the relation : $n_{d}\left(C_{n}\right)=n_{d}\left(P_{n-1}\right)+2(n-1)+\sum_{i=2}^{n-3} n_{d}\left(P_{n-i-2}\right)$.

Proof. Let $C_{n}$ be the cycle $v_{1}, v_{2}, \ldots, v_{n}, v_{1}$. Let $x_{1}$ denote the number of derived subgraphs of $C_{n}$ not containing $v_{1}$, then $x_{1}$ $=n_{d}\left(P_{n-1}\right)$. On the other hand, let $x_{2}$ denote the number of derived subgraphs which contain $v_{1}$. Such a derived subgraph contains a path $P_{i}$ of length $(i-1)$ that contains $v_{1}$ and a derived subgraph of path $P_{i}$ of order $(n-i-2)$, where $2 \leq i \leq n-1$. Then for fixed $i$ this number is $i n_{d}\left(P_{n-i-2}\right)$. Thus

$$
x_{2}=\sum_{i=2}^{n-3} i n_{d}\left(P_{n-i-2}\right)+(n-2)+(n-1)
$$

Moreover $C_{n}$ is a derived subgraph of itself, therefore $n_{d}\left(C_{n}\right)$ $=x_{1}+x_{2}+1$ so

$$
n_{d}\left(C_{n}\right)=n_{d}\left(P_{n-1}\right)+2(n-1)+\sum_{i=2}^{n-3} i n_{d}\left(P_{n-I-2}\right)
$$

Theorem 3. Let $G(n, n)$ be a bipartite graph with two partitioning sets $V_{1}$ and $V_{2}$, where $\left|V_{1}\right|=\left|V_{2}\right|=n$ and $\operatorname{deg}(v)=n$ - 1 for each $v \in V(G(n, n))$. Then the number of derived subgraphs of $G(n, n)$ ) is given by the relation: $n_{d}(G(n, n))=2^{2 n}+n+2-n 2^{n}-2^{n+1}$, and each edge

$u v \in E(G(n, n))$ is contained in exactly $2^{n-1}\left(2^{n-1}-1\right)$ derived subgraphs.

Proof. Let $V_{1}=v_{1}, v_{2}, \ldots, v_{n}$ and $V_{2}=u_{1}, u_{2}, \ldots, u_{n}$ where $u_{i} v_{i} \notin E(G(n, n))$ for each $i=1,2, \ldots, n$.

To form a derived subgraph, we take $S_{1} \cup S_{2}$ where $S_{i} \subset V_{i}$ for $i=1$, 2. If $\left|S_{1}\right| \geq 2$ and $\left|S_{2}\right| \geq 2$ then we get a derived subgraph.

If $\left|S_{1}\right|=1$, say, $S_{1}=v_{i}$ then $\phi \neq S_{2} \cup V_{2} \backslash\left\{u_{i}\right\}$. This shows that

$$
n_{d}(G(n, n))=\left(2^{n}-n-1\right)^{2}+1+2 n\left(2^{n}-1\right)
$$

Now we fix an $i=1,2, \ldots, n$. We count the number of derived subgraphs which contain the edge $v_{1} u_{i}$. Such derived subgraph will have the form $S_{1} \cup S_{2}$ where $v_{1} \in S_{1} \subset V_{1}$.

Again if $\left|S_{1}\right| \geq 2$ and $\left|S_{2}\right| \geq 2$ then we have a derived subgraph.

If, say $S_{1}=\left\{v_{1}\right\}$ then $u_{1} \notin S_{2}$. This shows that the number of derived subgraphs which contain $v_{i} u_{i}$ is equal to $\left(2^{n-1}-1\right)^{2}+2\left(2^{n-2}\right)-1=2^{2 n-2}-2^{n-1}$

\section{THE SERIAL DERIVED SUBGRAPH ALGORITHM}

In this Section, we introduce a serial derived subgraphs algorithm SDSA which calculates the number of derived subgraphs for a given graph $G$. The algorithm also determines the residual and non-residual edges. The parameters of the algorithm are :

$A[i, j] \quad$ : the adjacency matrix of $G$.

$S$ [i] $\quad$ : all of the subsets of $V(G)$.

$(i, j) \quad$ : the entry of the matrix $E(i, j)$ which is equal to the number of derived subgraphs that contain $v_{i} v_{j}$

total : the number of all derived subgraphs of $G$.

Let $G$ be a graph which has $n$ vertices and $m$ edges. We can represent the graph $G$ by the Adjacency-Graph class, where $a[i][j]$ is the entry element $(i, j)$ in the adjacency matrix $A$. The algorithm finds all subsets of the vertex set $V(G)$; then it checks if the current subset induces a derived subgraph or not. The algorithim finds the number of derived subgraphs that contain any edge $e \in E(G)$

Our main algorithm SDSA calls three procedures InitializeSubset, Get-Next-Subset and Check-Subset as follows:

Algorithm 1: A serial derived subgraphs algorithm $S D S A$

Input : $A[i][j]$ the adjacency matrix of $G$.

Output : ( total ) the number of all derived subgraphs of $G$. 


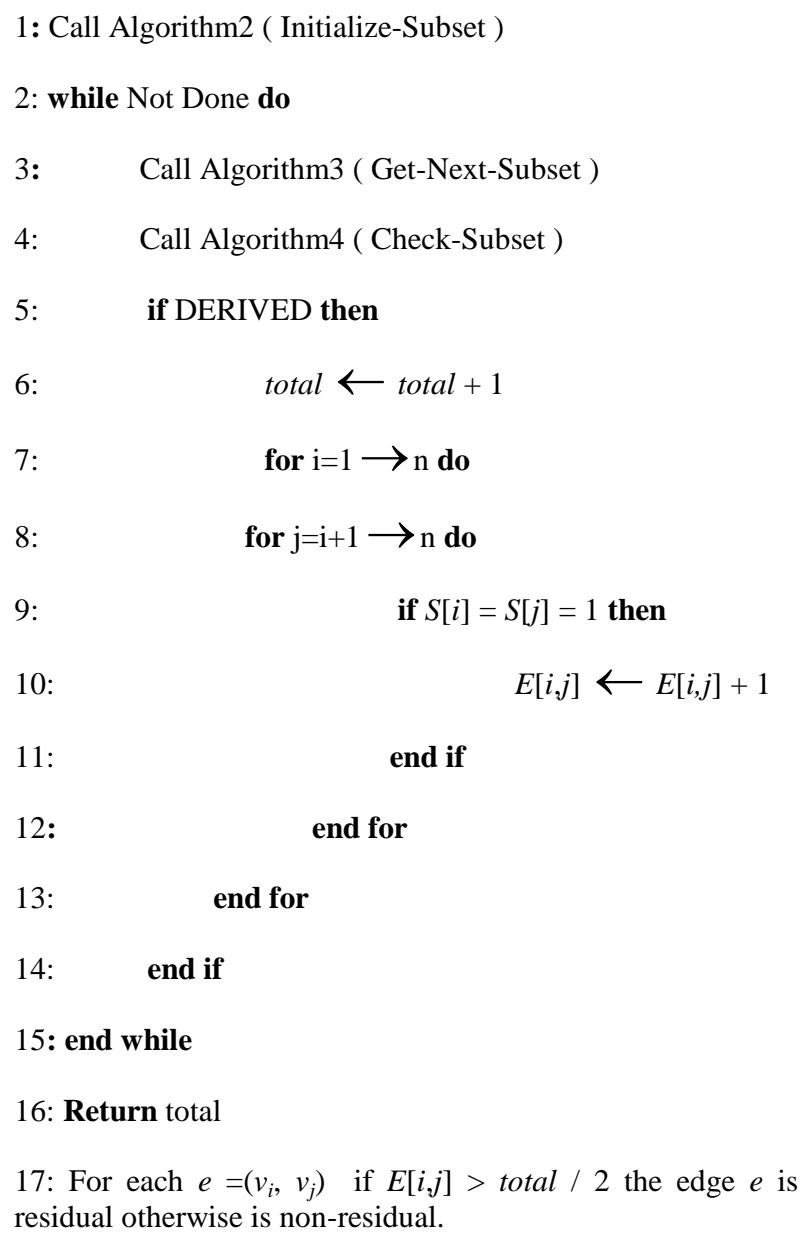

The Initialize-Subset procedure initializes the initial subset of $V(G)$ as array $S[j]=0$. The subgraph induced by the initial $S$ is the empty derived subgraph. We outline below the initialize-Subset procedure which considers the empty subgraph as the first derived one.

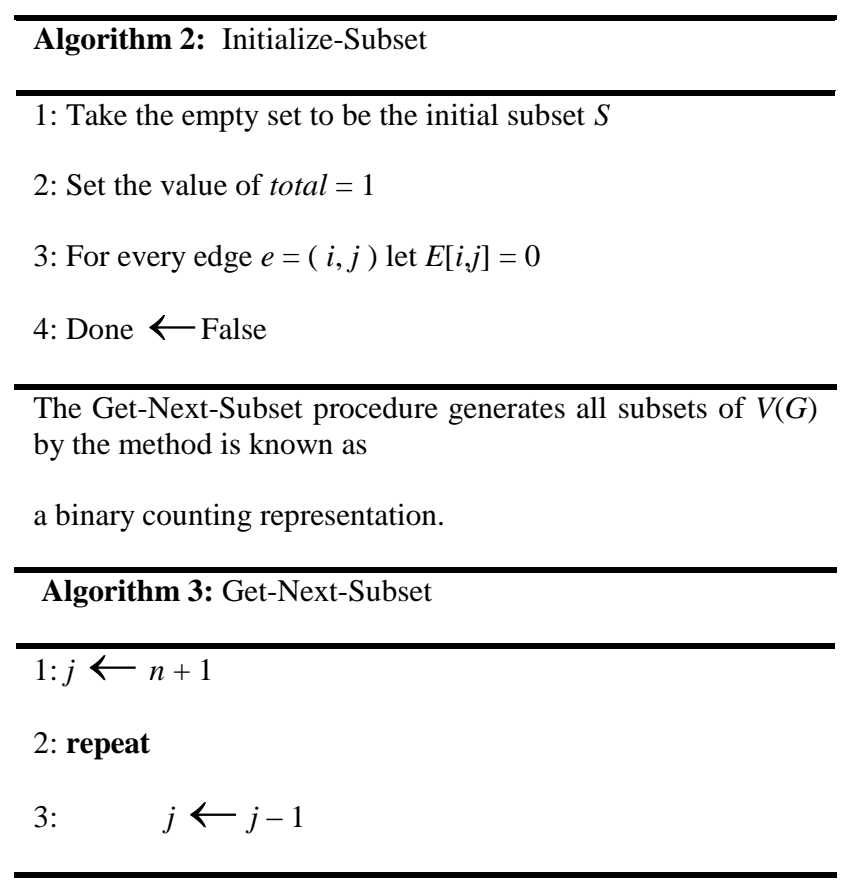

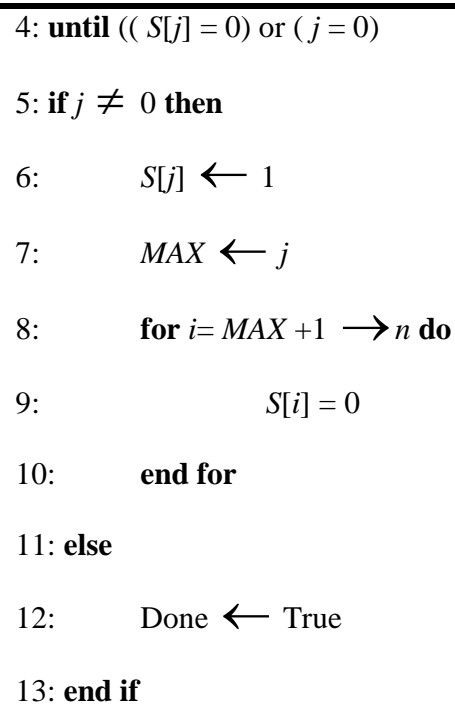

The Check-Subset verifies the current subset $S$ as a derived subgraph or not. A precise description of this process is the following.

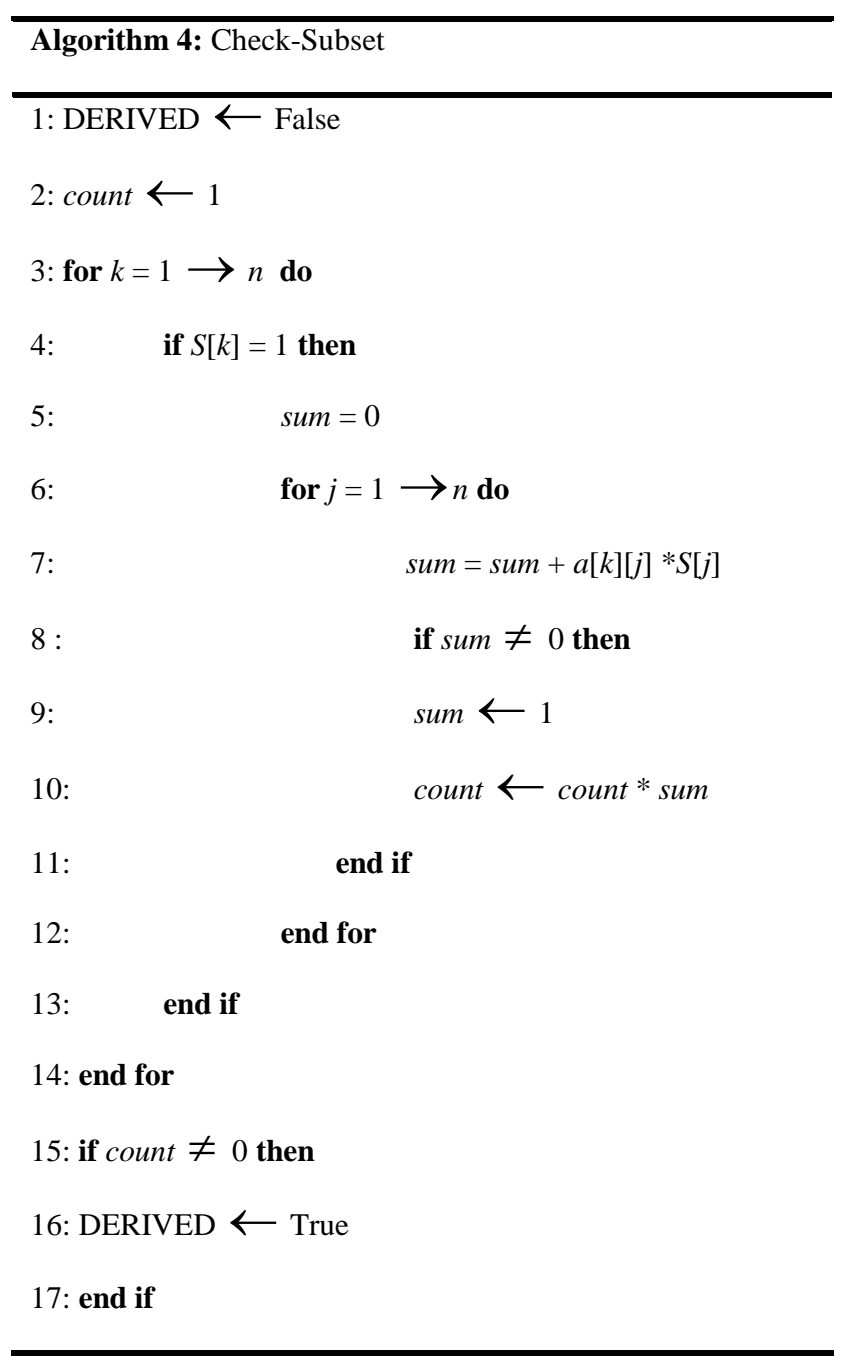




\section{4- COMPLEXITY ANALYSIS AND COMPUTATIONAL RESULTS}

The SDSA algorithm can be shown to run in $\mathrm{O}\left(n 2^{n}\right)$ time where $n$ is the number of vertices in the given graph $G$. There are $2^{n}$ subsets of $V(G)$. We check every one by calling Algorithm 4 (Check-Subset). The Algorithm 3 ( Get-NextSubset ) requires $\mathrm{O}(n)$ time. We need exactly $2^{n}-1$ calls of Algorithm 3 (Get-Next-Subset), each one runs in $\mathrm{O}(n)$ time. Then the total running time of SDSA algorithm is $\mathrm{O}\left(n 2^{n}\right)$ sequential time.

The algorithm describe in Section 3 has been experimentally implemented. In this Section, the numerical experiments are presented. It must be mentioned that the computational results demonstrates the number of derived subgraphs and its residual edges for some special graphs.

Our numerical experiments were performed on a PC with $2.000 \mathrm{MHz}$ Pentium 4 processor, RAM $512 \mathrm{Mb}$ and windows $\mathrm{XP}$ operating system. Our implementation was done under the $\mathrm{C}$ environment.

Columns of the following tables contain problems size $\boldsymbol{n}$ and $\boldsymbol{m}$, number of all derived subgraphs $N o \_D S$, number of all non-residual edges No_NRE and the running time CPU Time (secs.).

Table 1. Derived subgraphs for the path graph $P_{n}$

\begin{tabular}{|c|c|c|c|}
\hline$n$ & No_DS & No_NRE & $\begin{array}{c}\text { CPU Time } \\
\text { (secs.) }\end{array}$ \\
\hline 2 & 2 & 1 & 0.000181 \\
\hline 4 & 7 & 2 & 0.000367 \\
\hline 6 & 21 & 5 & 0.001085 \\
\hline 8 & 65 & 7 & 0.004173 \\
\hline 10 & 200 & 9 & 0.018365 \\
\hline 12 & 616 & 11 & 0.079329 \\
\hline 14 & 1897 & 13 & 0.352686 \\
\hline 16 & 5842 & 15 & 1.74612 \\
\hline 18 & 17991 & 17 & 7.962174 \\
\hline 20 & 55405 & 19 & 33.045809 \\
\hline
\end{tabular}

Table 2. Derived subgraphs for the cyclic graph $C_{n}$

\begin{tabular}{|c|c|c|c|}
\hline $\boldsymbol{n}$ & No_DS & No_NRE & $\begin{array}{c}\text { CPU Time } \\
\text { (secs.) }\end{array}$ \\
\hline 4 & 10 & 4 & 0.000356 \\
\hline 6 & 29 & 6 & 0.001080 \\
\hline 8 & 90 & 8 & 0.004166 \\
\hline 10 & 277 & 10 & 0.17741 \\
\hline
\end{tabular}

\begin{tabular}{|c|c|c|c|}
\hline 12 & 853 & 12 & 0.079052 \\
\hline 14 & 2627 & 14 & 0.355268 \\
\hline 16 & 8090 & 16 & 1.593558 \\
\hline 18 & 24914 & 18 & 7.371244 \\
\hline 20 & 76725 & 20 & 33.170715 \\
\hline
\end{tabular}

Table 3. Derived subgraphs for the complete graph $\mathbf{K}_{\mathbf{n}}$

\begin{tabular}{|c|c|c|c|}
\hline$n$ & No_DS & No_NRE & $\begin{array}{c}\text { CPU Time } \\
\text { (secs.) }\end{array}$ \\
\hline 4 & 12 & 6 & 0.000358 \\
\hline 6 & 58 & 15 & 0.001103 \\
\hline 8 & 248 & 28 & 0.004330 \\
\hline 10 & 1014 & 45 & 0.018671 \\
\hline 12 & 4084 & 66 & 0.084653 \\
\hline 14 & 16370 & 91 & 0.387049 \\
\hline 16 & 65520 & 120 & 1.754086 \\
\hline 18 & 262126 & 153 & 8.062705 \\
\hline 20 & 1048556 & 190 & 37.126845 \\
\hline
\end{tabular}

Table 4. Derived subgraphs for the complete bipartite graph $K_{m, n}$

\begin{tabular}{|c|c|c|c|c|}
\hline $\boldsymbol{m}$ & $\boldsymbol{n}$ & No_DS & No_NRE & $\begin{array}{c}\text { CPU Time } \\
\text { (secs.) }\end{array}$ \\
\hline 1 & 1 & 2 & 1 & 0.000185 \\
\hline 2 & 2 & 10 & 4 & 0.000365 \\
\hline 3 & 3 & 50 & 9 & 0.001098 \\
\hline 4 & 4 & 226 & 16 & 0.004296 \\
\hline 5 & 5 & 962 & 25 & 0.018593 \\
\hline 6 & 6 & 3970 & 36 & 0.084717 \\
\hline 7 & 7 & 16130 & 49 & 0.385195 \\
\hline 8 & 8 & 65026 & 64 & 1.753573 \\
\hline 9 & 9 & 261122 & 81 & 8.358592 \\
\hline 10 & 10 & 1046530 & 100 & 37.569930 \\
\hline
\end{tabular}




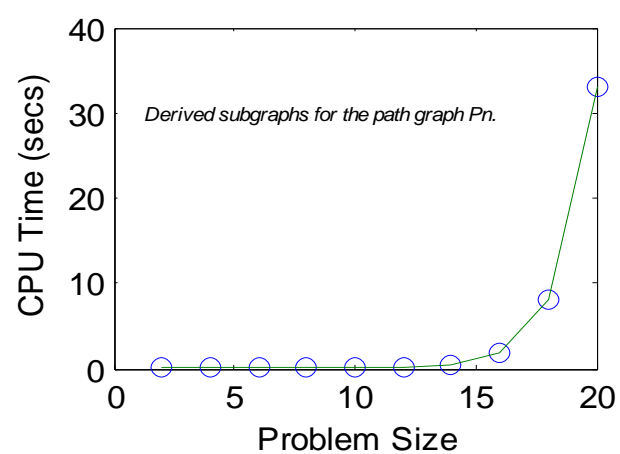

Fig.3: Executable time for $P_{n}$ graph by $S D S A$

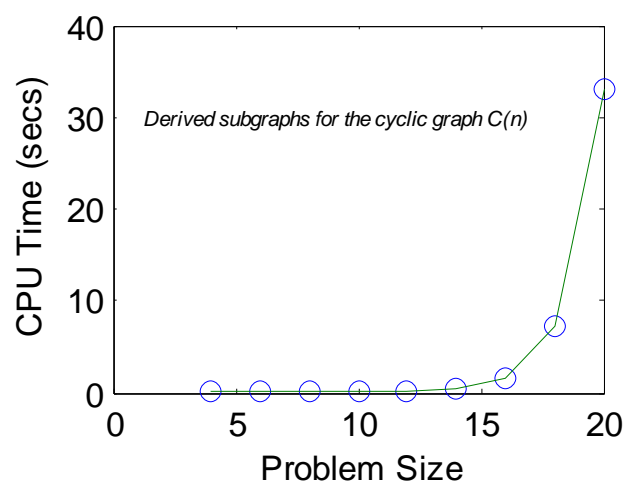

Figure 4. Executable time for $C_{n}$ graph by $S D S A$

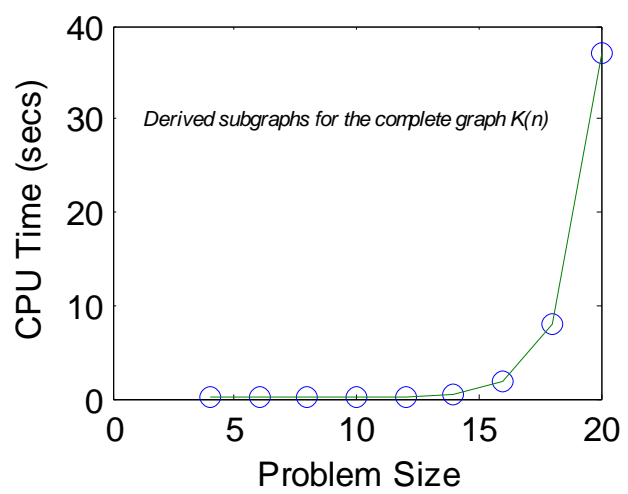

Figure 5. Executable time for $K_{n}$ graph by $S D S A$

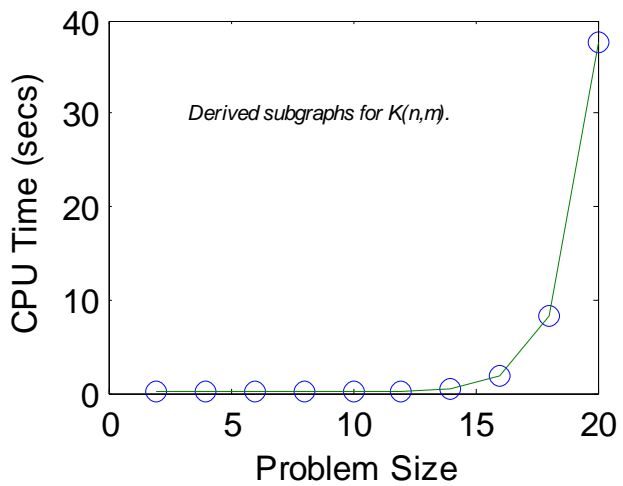

Figure 6. Executable time for $K_{n, m}$ graph by SDSA

From the above Tables, we can note that every graph for any size contains a non-residual edge (Conjecture 3 ) while from the above Plots, we can see that the running time of the algorithm SDSA increases as an exponential time in the problem size $n$.

\section{CONCLUDING REMARKS}

In this work we proved some theorems which calculate the number of derived subgraphs for some special graphs. We also presented a new algorithm SDSA that calculates the number of derived subgraphs for a given graph $G$ and determines the residual and non-residual edges. Finally, we introduced a computational study which supports our results. Our algorithm SDSA has $\mathrm{O}\left(n 2^{n}\right)$ sequential time so there is room for its further improvement as an sequential or parallel algorithm. These possible improvements will be the subject of our future work.

\section{REFERENCES}

[1] I. Rival (Ed.), Graphs And Order, Reidel, DordrechtBoston,(1985), p.25.

[2] R. P. Stanley, Enumerative Combinatorics, vol. I, Wadsworth \& Broks/Cole, Belmont, CA, (1986).

[3] B. Poonen, Union-Closed Families, J. Combin. Theory, A 59 (1992), 253-268.

[4] M. H. El-Zahar, A Graph-Theoretic Version Of The Union-Closed Sets Conjecture, J.Graph Theory 26 (1997), no. 3, 155-163.

[5] B. Llano, J. Montellano-Ballesteros, E. Rivera-Campo and R. Strauz " On Conjecture of Frankl and El-Zahar" J. Graph Theory 57: 344-352 (2008).

[6] G. Chartrand and L. Lesniak " Graphs \& Digraphs" (third edition) Chaman \& Hall, London, (1996) . 